\begin{tabular}{|c|l|}
\hline Title & A tomki anomaly and dark matter in a radiative seesaw model with gauged B : L symmetry \\
\hline Author(s) & Seto, Osamu; Shimomura, Takashi \\
\hline Citation & $\begin{array}{l}\text { Physical review D, 95(9), 095032-1-095032-9 } \\
\text { https://doi.org/10.1103/PhysRevD.95.095032 }\end{array}$ \\
\hline Issue Date & 2017-05-31 \\
\hline Doc URL & http://hdl.handle.net/2115/67012 \\
\hline Rights & @2017 A merican Physical Society \\
\hline Type & article \\
\hline File Information & PhysRevD.95.095032.pdf \\
\hline
\end{tabular}

Instructions for use 


\title{
Atomki anomaly and dark matter in a radiative seesaw model with gauged $B-L$ symmetry
}

\author{
Osamu Seto ${ }^{1,2, *}$ and Takashi Shimomura ${ }^{3, \dagger}$ \\ ${ }^{1}$ Institute for International Collaboration, Hokkaido University, Sapporo 060-0815, Japan \\ ${ }^{2}$ Department of Physics, Hokkaido University, Sapporo 060-0810, Japan \\ ${ }^{3}$ Faculty of Education, Miyazaki University, Miyazaki 889-2192, Japan
}

(Received 19 March 2017; published 31 May 2017)

\begin{abstract}
Motivated by recently reported anomalies in a decay of an excited state of beryllium by the Atomki Collaboration, we study a radiative seesaw model with gauged $B-L$ symmetry and a $Z_{2}$ parity. Assuming that the anomalies originate from the decay of the $B-L$ gauge boson followed by the nuclear decay, the mass of the lightest right-handed neutrino or the dark matter candidate can be determined below $10 \mathrm{GeV}$. We show that for this mass range, the model can explain the anomalies in the beryllium decay and the relic dark matter abundance consistent with neutrino masses. We also predict its spin-independent cross section in direct detection experiments for this mass range.
\end{abstract}

DOI: 10.1103/PhysRevD.95.095032

\section{INTRODUCTION}

The Standard Model (SM) of particle physics has been explaining almost all of experimental results including recent LHC data. Despite its enormous success, some phenomena are left unexplained in the SM. One such phenomena is neutrino oscillations, which result in nonzero and tiny neutrino masses as well as flavor mixing. Another one is the existence of dark matter (DM). Since neutrinos are massless and no candidates exist in the SM, these phenomena are clear evidences of new physics beyond the SM.

Several mechanisms have been proposed to explain the tininess of neutrino mass. The most popular mechanism is the so-called type I seesaw mechanism [1-4]. In the mechanism, right-handed (rh) neutrinos with heavy Majorana masses are introduced to the SM, and the tiny neutrino masses can be explained by a suppression of their heavy mass. There are other types of seesaw mechanisms, type II [5-7], type III [8], and radiative models [9-11]. In radiative seesaw mechanisms, a discrete parity is generally imposed to the SM so that neutrinos can not have tree-level masses or Yukawa interactions (For pioneering works, see, e.g., Refs. $[12,13])$. Then, neutrino masses are generated at loop-level in which new scalars and/or fermions propagate. The masses generated are suppressed by the masses of the new particles in the loop and a loop factor. Tininess of neutrino masses is explained in this sense. In addition to the generation of neutrino mass, the radiative seesaw mechanisms have another virtue. The lightest particle with odd parity becomes stable due to the discrete parity. Such a stable particle can be a good candidate for the DM. In fact, many radiative seesaw models can predict the correct DM

*seto@particle.sci.hokudai.ac.jp

†shimomura@cc.miyazaki-u.ac.jp abundance. Thus, the two phenomena mentioned above can be explained simultaneously.

Recently, the Atomki Collaboration has reported anomalies in the distributions of the invariant mass and the opening angle of an emitted electron-positron pair from the decay of an excited state of beryllium $\left({ }^{8} \mathrm{Be}\right)$ into its ground state [14]. They claimed that the deviation from a standard nuclear physics interpretation reaches to $6.8 \sigma$, and hence, the deviation is probably not a nuclear physics origin. We refer these anomalies to the Atomki anomalies. One of the possibilities to explain the anomalies is the subsequent decay of an unknown particle produced in the ${ }^{8}$ Be decay. The Atomki Collaboration assumed a new boson particle with spin-parity $J^{\pi}=1^{+}$and determined its mass as $m=16.70 \pm 0.35$ (stat) \pm 0.5 (syst) $\mathrm{MeV}$ from their data. It is natural to consider that the boson acquires such a light mass from a spontaneous breakdown of a symmetry. Then, a fundamental scale of the nature can be determined. In $[15,16]$, the authors showed the Atomki anomalies can be explained by a gauge boson in classes of models with gauged baryon $(B)$ and baryon minus lepton $(B-L)$ symmetry. These gauge symmetries are one of the minimal extensions of the SM and have been extensively studied in terms of various motivations. There are also studies to explain the Atomki anomalies in other gauge symmetries [17,18], with an axial vector [19] and pseudoscalars [20]. Implications on the DM have also been studied [21-23].

In this paper, we study the implications of the Atomki anomalies in a radiative seesaw model with gauged $B-L$ symmetry proposed by us [24]. We find parameter values consistent with experiments by taking into account the neutrino mass, the Higgs mass as well as the new boson mass. Then, we predict the spin-independent cross section consistent with the dark matter abundance. Various radiative seesaw mechanism with $Z_{2}$ parity and gauged 
$U(1)_{B-L}$ symmetries have been proposed [24-36]. Our following discussion and results would be applicable once one tries to address the Atomki anomalies in such a model, because the required cross section and the mass determine the scale of the $U(1)_{B-L}$ symmetry.

The rest of this paper is organized as follows. In Sec. II, we explain our model including brief review of neutrino masses. We show the interaction Lagrangian of the gauge boson with SM fermions as well as constraints for the Atomki anomalies to be explained. Then, parameters and masses consistent with experimental constraints are derived in Sec. III. In Sec. IV, the spin-independent cross section is predicted for the parameter values derived in Sec. III. We summarize our study in Sec. V.

\section{MODEL}

We explain our model proposed in Ref. [24]. The SM is extended by imposing the gauged $U(1)_{B-L}$ symmetry and a $Z_{2}$ parity, and also introducing two scalar particles and three right-handed neutrinos, $N_{R}$. One of the scalar particles, $S$, is a SM singlet and responsible for $B-L$ symmetry breaking. The other one, $\eta$, has the same quantum charge as the SM Higgs $\Phi$ and is related with the generation of neutrino masses. The scalar $\eta$ and the rh neutrinos $N_{R}$ are $Z_{2}$ odd while other particles are $Z_{2}$ even. The charge assignment of the particles is summarized in Table I. Here, $Q^{i}, d_{R}^{i}, u_{R}^{i}$, and $L^{i}, e_{R}^{i}$ are the left-handed (lh) and the right-handed quarks and leptons, respectively. Latin and Greek indices denote generation and flavor of fermions.

First, we briefly review the neutrino mass in our model. The interaction Lagrangian for the generation of neutrino mass is given by

$$
\mathcal{L}_{\text {int }}=\mathcal{L}_{N}-V(\Phi, \eta, S),
$$

where the Yukawa interactions are given by

$$
\mathcal{L}_{N}=g_{i \alpha} \bar{L} i \tilde{\eta} N_{R}^{\alpha}-\frac{Y_{R}^{\alpha}}{2} S \overline{\left(N_{R}^{\alpha}\right)^{c}} N_{R}^{\alpha}+\text { H.c., }
$$

and the scalar potential is given by

$$
\begin{aligned}
V(\Phi, \eta, S)= & \mu_{1}^{2}|\Phi|^{2}+\mu_{2}^{2}|\eta|^{2}+\mu_{S}^{2}|S|^{2}+\lambda_{1}|\Phi|^{4}+\lambda_{2}|\eta|^{4} \\
& +\lambda_{3}|\Phi|^{2}|\eta|^{2}+\lambda_{4}\left|\Phi^{\dagger} \eta\right|^{2}+\frac{\lambda_{5}}{2}\left[\left(\Phi^{\dagger} \eta\right)^{2}+\text { H.c. }\right] \\
& +\lambda_{S}|S|^{4}+\tilde{\lambda}|\Phi|^{2}|S|^{2}+\lambda|\eta|^{2}|S|^{2}
\end{aligned}
$$

TABLE I. The charge assignment of fields.

\begin{tabular}{lccccccccc}
\hline \hline & $Q^{i}$ & $d_{R}^{i}$ & $u_{R}^{i}$ & $L^{i}$ & $e_{R}^{i}$ & $\Phi$ & $\eta$ & $S$ & $N_{R}^{\alpha}$ \\
\hline$S U(3)_{C}$ & 3 & 3 & 3 & 1 & 1 & 1 & 1 & 1 & 1 \\
$S U(2)_{W}$ & 2 & 1 & 1 & 2 & 1 & 2 & 2 & 1 & 1 \\
$U(1)_{Y}$ & $1 / 6$ & $-1 / 3$ & $+2 / 3$ & $1 / 2$ & -1 & $1 / 2$ & $1 / 2$ & 0 & 0 \\
$U(1)_{B-L}$ & $1 / 3$ & $1 / 3$ & $1 / 3$ & -1 & -1 & 0 & 0 & +2 & -1 \\
$Z_{2}$ & + & + & + & + & + & + & - & + & - \\
\hline \hline
\end{tabular}

with $\tilde{\eta}=i \sigma_{2} \eta^{*}$. The summation over repeated indices should be understood. Because of the $Z_{2}$ parity, neutrinos can not have Yukawa couplings with the Higgs field $\Phi$; instead, they can have those with $\eta$. After the Higgs and the scalar $S$ develop vacuum expectation values (VEVs),

$$
\langle\Phi\rangle=\frac{1}{\sqrt{2}}\left(\begin{array}{l}
0 \\
v
\end{array}\right), \quad\langle S\rangle=\frac{v_{S}}{\sqrt{2}},
$$

where $v=246 \mathrm{GeV}$, the masses of neutrinos are generated via a one-loop diagram in which $N_{R}$ and $\eta$ propagate, and expressed as

$$
m_{\nu_{L}}^{i j} \simeq \frac{\lambda_{5}}{8 \pi^{2}} g_{i \alpha} Y_{R}^{\alpha} g_{\alpha j}^{T}\left(\frac{v}{m_{\eta}}\right)^{2} v_{S}
$$

More details can be found in Ref. [24].

Next, we consider the gauge sector. The relevant Lagrangian to explain the Atomki anomalies is given by

$$
\mathcal{L}=\mathcal{L}_{\text {gauge, int }}+\mathcal{L}_{\text {gauge,kin }}
$$

where

$\mathcal{L}_{\text {gauge,int }}=g_{1} \hat{B}_{\mu} J_{1}^{\mu}+g_{2} \hat{W}_{\mu}^{a} J_{2}^{a \mu}+\epsilon_{X} e \hat{X}_{\mu} J_{X}^{\mu}$,

$\mathcal{L}_{\text {gauge,kin }}=-\frac{1}{4} \hat{B}_{\mu \nu} \hat{B}^{\mu \nu}-\frac{1}{4} \hat{W}_{\mu \nu}^{a} \hat{W}^{a \mu \nu}-\frac{1}{4} \hat{X}_{\mu \nu} \hat{X}^{\mu \nu}+\frac{\epsilon}{2} \hat{B}_{\mu \nu} \hat{X}^{\mu \nu}$.

Here, $\hat{B}, \hat{W}^{a}, \hat{X}$ represent $U(1)_{Y}, S U(2)_{W}$, and $U(1)_{B-L}$ gauge bosons in the interaction basis, in which $a$ being $S U(2)$ index, while $\hat{B}^{\mu \nu}, \hat{W}^{a \mu \nu}, \hat{X}^{\mu \nu}$ are their field strengths, respectively. The coupling constants and the currents of $U(1)_{Y}, S U(2)_{W}$, and $U(1)_{B-L}$ are denoted as $g_{1}, g_{2}$, and $\epsilon_{X} e$, and $J_{1}^{\mu}, J_{2}^{a \mu}$, and

$J_{X}^{\mu}=\frac{1}{3} \overline{u^{i}} \gamma^{\mu} u^{i}+\frac{1}{3} \overline{d^{i}} \gamma^{\mu} d^{i}-\overline{e^{i}} \gamma^{\mu} e^{i}-\overline{\nu^{i}} \gamma^{\mu} \nu^{i}-\overline{N_{R}^{i}} \gamma^{\mu} N_{R}^{i}$.

Note that the gauge coupling constant of $U(1)_{B-L}$ is normalized by the electric charge of electron for convenience. The kinetic mixing parameter is denoted as $\epsilon$.

After the electroweak and the $B-L$ symmetries are broken, the interaction Lagrangian of the neutral gauge bosons in the mass basis is given as

$$
\begin{aligned}
\mathcal{L}_{\text {gauge,int }}= & e A_{\mu} J_{e m}^{\mu}+Z_{\mu}\left[g_{2}\left(c_{\chi}-\varepsilon s_{W} s_{\chi}\right) J_{N C}^{\mu}+\varepsilon c_{W} s_{\chi} J_{e m}^{\mu}\right. \\
& \left.+\varepsilon_{X} e s_{\chi} J_{X}^{\mu}\right]+X_{\mu}\left[\varepsilon_{X} e c_{\chi} J_{X}^{\mu}+\varepsilon e c_{W} c_{\chi} J_{e m}^{\mu}\right. \\
& \left.-g_{2}\left(s_{\chi}+\varepsilon s_{W} c_{\chi}\right) J_{N C}^{\mu}\right] \\
\mathcal{L}_{\text {gauge,kin }}= & -\frac{1}{4} F_{\mu \nu} F^{\mu \nu}-\frac{1}{4} Z_{\mu \nu} Z^{\mu \nu}+\frac{1}{2} m_{Z}^{2} Z_{\mu} Z^{\mu}-\frac{1}{4} X_{\mu \nu} X^{\mu \nu} \\
& +\frac{1}{2} m_{X}^{2} X_{\mu} X^{\mu},
\end{aligned}
$$


where $A_{\mu}\left(F_{\mu \nu}\right)$ and $Z_{\mu}\left(Z_{\mu \nu}\right)$ represent the SM photon and the $Z$ boson (and their field strengths), respectively. The currents $J_{e m}^{\mu}$ and $J_{N C}^{\mu}$ are the same as those in the SM. The dimensionless parameters $\varepsilon$ and $\varepsilon_{X}$ are defined as

$$
\varepsilon=\epsilon r, \quad \varepsilon_{X}=\epsilon_{X} r
$$

with $r=\left(1-\epsilon^{2}\right)^{-1 / 2}$. The weak mixing angle is denoted as $s_{W}=\sin \theta_{W}\left(c_{W}=\cos \theta_{W}\right)$, and the mixing angle of the gauge bosons due to the kinetic mixing, $s_{\chi}=\sin \chi\left(c_{\chi}=\cos \chi\right)$, is defined by

$$
\tan 2 \chi=\frac{-m_{\hat{Z}}^{2} q}{\left(1-q^{2}\right) m_{\hat{Z}}^{2}-m_{\hat{X}}^{2} r^{2}},
$$

with $q=-\varepsilon s_{W}$, and

$$
\begin{aligned}
& m_{\hat{Z}}=\frac{1}{2} \sqrt{g_{1}^{2}+g_{2}^{2}} v, \\
& m_{\hat{X}}=2 \epsilon_{X} e v_{s} .
\end{aligned}
$$

The masses of the gauge bosons are given as

$$
\begin{aligned}
m_{Z}^{2} & =\frac{1}{2}\left[m_{\hat{Z}}^{2}\left(1+q^{2}\right)+m_{\hat{X}}^{2} r^{2}+\sqrt{D}\right], \\
m_{X}^{2} & =\frac{1}{2}\left[m_{\hat{Z}}^{2}\left(1+q^{2}\right)-m_{\hat{X}}^{2} r^{2}+\sqrt{D}\right], \\
D & =\left(m_{\hat{Z}}^{2}\left(1+q^{2}\right)+m_{\hat{X}}^{2} r^{2}\right)^{2}-4 m_{\hat{Z}}^{2} m_{\hat{X}}^{2} r^{2} .
\end{aligned}
$$

For $|\varepsilon|,\left|\varepsilon_{X}\right| \ll 1$, the mixing angle can be approximated

$$
s_{\chi} \simeq-\epsilon s_{W}, \quad c_{\chi} \simeq 1 .
$$

Then, the interaction Lagrangian and the gauge boson masses are given by

$$
\begin{gathered}
\mathcal{L}_{\text {gauge, int }}=e A_{\mu} J_{e m}^{\mu}+g_{2} Z_{\mu} J_{N C}^{\mu} \\
+e X_{\mu}\left[\varepsilon_{X} J_{X}^{\mu}+\varepsilon c_{W} J_{e m}^{\mu}\right]+\mathcal{O}\left(\epsilon^{2}, \epsilon \epsilon_{X}\right), \\
m_{Z}^{2} \simeq m_{\hat{Z}}^{2}+\mathcal{O}\left(\epsilon^{2}\right), \\
m_{X}^{2} \simeq m_{\hat{X}}^{2}+\mathcal{O}\left(\epsilon^{2}\right) .
\end{gathered}
$$

From Eq. (15a), the resulting coupling constants of the SM fermions to the $X$ boson are

$$
\begin{aligned}
& \varepsilon_{u}=\frac{1}{3} \varepsilon_{X}+\frac{2}{3} \varepsilon c_{W}, \\
& \varepsilon_{d}=\frac{1}{3} \varepsilon_{X}-\frac{1}{3} \varepsilon c_{W}, \\
& \varepsilon_{\nu}=-\varepsilon_{X},
\end{aligned}
$$

$$
\varepsilon_{e}=-\varepsilon_{X}-\varepsilon c_{W}
$$

The coupling constants of an up and a down quark can be translated into those of a proton and a neutron as

$$
\varepsilon_{p}=2 \varepsilon_{u}+\varepsilon_{d}, \quad \varepsilon_{n}=\varepsilon_{u}+2 \varepsilon_{d} .
$$

To explain the Atomki anomalies, $\left|\varepsilon_{n}\right|$ and $\left|\varepsilon_{p}\right|$ are required to satisfy [16]

$$
\begin{gathered}
\left|\varepsilon_{n}\right|=(2-10) \times 10^{-3}, \\
\left|\varepsilon_{p}\right| \lesssim 1.2 \times 10^{-3} .
\end{gathered}
$$

On the other hand, these coupling constants are constrained by several experiments, i.e., the dark photon searches in neutral pion decays, beam dump searches, neutrinoelectron scatterings. The constraints as well as the signal requirements are summarized as [16]

$$
\begin{aligned}
\left|\varepsilon_{n}\right| & =\left|\varepsilon_{X}\right|=(2-10) \times 10^{-3}, \\
\left|\varepsilon_{p}\right| & =\left|\varepsilon_{X}-\varepsilon c_{W}\right| \lesssim 1.2 \times 10^{-3} \\
\left|\varepsilon_{e}\right| & =(0.2-1.4) \times 10^{-3} \\
\sqrt{\left|\varepsilon_{e} \varepsilon_{\nu}\right|} & \lesssim 3 \times 10^{-4} .
\end{aligned}
$$

Note that in our model, $\varepsilon_{\nu}=-\varepsilon_{n}$ and hence, the above experimental constraints are not satisfied. However, it is possible to evade the constraint by further extensions. One of such successful extensions is to introduce pairs of vectorlike leptons [16] whose SM gauge charges are the same while the $B-L$ charge is opposite. Because of the opposite $B-L$ charge, the mixing between the lh neutrinos and the vectorlike neutrinos can suppress the lightest neutrino coupling to $X$ so that the constraints can be satisfied. This extension can be applied to our model and make the lightest neutrino to be neutralized to the $X$ boson. For concreteness, we consider one pair of the vectorlike leptons $L_{L}^{4}=\left(\nu_{L}^{4}, e_{L}^{4}\right)^{T}, L_{R}^{4}=$ $\left(\nu_{R}^{4}, e_{R}^{4}\right)^{T}$ and $E_{L}^{4}, E_{R}^{4}$ that are $S U(2)_{W}$ doublet and singlet, respectively. We assign the even parity of $Z_{2}$ to these leptons. Then, the mass term of the neutrinos after the symmetry breaking is given by

$\mathcal{L}_{\text {mass }}=-\frac{1}{2} m_{M} \overline{N_{R}^{c}} N_{R}-M_{4 L} \overline{\nu_{R}^{4}} \nu_{L}-M_{L} \overline{\nu_{L}^{4}} \nu_{R}^{4}+$ H.c.,

where $m_{M}$ and $M_{4 L}$ are proportional to $v_{S}$, and $M_{L}$ is a Dirac mass. It is important to note that the rh neutrinos can not mix with the other neutrinos due to the $Z_{2}$ parity and hence, be taken as mass eigenstates. The second and the third terms of Eq. (20) can be casted as $\overline{\psi_{L}^{\nu}} M_{\nu} \psi_{R}^{\nu}+$ H.c., where 


$$
\begin{aligned}
& M_{\nu}=\left(\begin{array}{ll}
0 & M_{4 L} \\
0 & M_{L}
\end{array}\right), \\
& \psi_{L}^{\nu}=\left(\begin{array}{c}
\nu_{L} \\
\nu_{L}^{4}
\end{array}\right), \\
& \psi_{R}^{\nu}=\left(\begin{array}{c}
N_{R} \\
\nu_{R}^{4}
\end{array}\right) .
\end{aligned}
$$

Diagonalizing Eq. (21a), we obtain the one Dirac state with mass $\sqrt{M_{L}^{2}+M_{4 L}^{2}}$ and one massless state. The latter state can be express as

$$
\frac{1}{\sqrt{M_{L}^{2}+M_{4 L}^{2}}}\left(-M_{L} \nu+M_{4 L} \nu_{L}^{4}\right),
$$

and its coupling to the $X$ boson is given by

$$
\epsilon_{\nu}=-\epsilon_{X} \cos 2 \theta_{\nu},
$$

where $\tan \theta_{\nu}=M_{4 L} / M_{L}$. Thus, the lightest neutrino can be neutralized by choosing $M_{4 L} \simeq M_{L}$. Generalization of $N$ pairs of the vectorlike leptons is straightforward and that allows new vector lepton to be heavier while the lightest neutrino is kept neutralized.

\section{PARAMETER VALUES}

In this section, we show parameter values in the model taking into account the $X$ gauge boson mass and couplings.

Firstly, the VEV of $S$ can be determined since the mass of the gauge boson (15c) should be $m_{X}=16.70 \pm$ 0.35 (stat) \pm 0.5 (syst) MeV [14],

$$
v_{s}=13.78\left(\frac{2 \times 10^{-3}}{\left|\varepsilon_{X}\right|}\right) \mathrm{GeV} \text {, }
$$

for the central value of $m_{X}$. We normalize $\left|\varepsilon_{X}\right|$ by $2 \times 10^{-3}$ as a reference value, which corresponds to the lower bound in Eq. (19a). On the other hand, the Higgs mass is given roughly by $\sqrt{2 \lambda_{1}} v$ where $\lambda_{1}$ is the quartic coupling in Eq. (3). Therefore, the quartic coupling $\lambda_{1}$ must be 0.130 to reproduce the Higgs mass $125 \mathrm{GeV}$. Using Eq. (24), the ratio of the VEVs, $\tan \beta$, and the mixing angle between $Z_{2}$ even scalars, $\alpha$, defined in Ref. [24] are expressed as

$$
\begin{aligned}
\tan \beta & =5.60 \times 10^{-2}\left(\frac{2 \times 10^{-3}}{\left|\varepsilon_{X}\right|}\right), \\
\alpha & \simeq 10^{-4}\left(\frac{\tilde{\lambda}}{4.65 \times 10^{-4}}\right)\left(\frac{0.130}{\lambda_{1}}\right)\left(\frac{2 \times 10^{-3}}{\left|\varepsilon_{X}\right|}\right),
\end{aligned}
$$

where $\tilde{\lambda}$ and $\lambda_{1}$ are normalized by reference values, respectively.
Then, the masses of the $Z_{2}$ even lighter scalar $H$ and $N_{R}$ are parametrized by

$$
\begin{aligned}
m_{H} & =19.5\left(\frac{\lambda_{s}}{1}\right)^{1 / 2}\left(\frac{2 \times 10^{-3}}{\left|\varepsilon_{X}\right|}\right) \mathrm{GeV}, \\
m_{N_{R}} & =9.75\left(\frac{Y_{R}}{1}\right)\left(\frac{2 \times 10^{-3}}{\left|\varepsilon_{X}\right|}\right) \mathrm{GeV},
\end{aligned}
$$

where $\lambda_{s}$ and $Y_{R}$ is a quartic coupling in the scalar potential and the couplings of rh neutrinos to $S$, respectively. One can see from Eqs. (26) that the masses of $H$ and $N_{R}$ are less than 20 and $10 \mathrm{GeV}$, respectively, when we require $\lambda_{s}, Y_{R} \leq 1$. These mass ranges are a direct consequence of the light gauge boson because the masses are proportional to $v_{S}$. Since the other $Z_{2}$ odd particle, $\eta$, should be heavier than $\mathrm{TeV}$ to give tiny neutrino masses, the lightest rh neutrino is the DM candidate in our model.

In the end, the lh neutrino masses given in Eq. (5) are parametrized as

$m_{\nu_{L}} \sim 0.10\left(\frac{\lambda_{5} g_{i \alpha}^{2}}{5.7 \times 10^{-9}}\right)\left(\frac{Y_{R}}{1}\right)\left(\frac{v / m_{\eta}}{0.1}\right)\left(\frac{2 \times 10^{-3}}{\left|\varepsilon_{X}\right|}\right) \mathrm{eV}$.

Adjusting $\lambda_{5}$ and $g_{i \alpha}$, the lh neutrino masses can be taken to the correct order, $0.1 \mathrm{eV}$, without conflicting other constraints. As shown in the next section, direct detection searches of the DM constrains $\alpha$ to be as small as $\mathcal{O}\left(10^{-4}\right)$, and hence, $\tilde{\lambda}$ should be smaller than $\mathcal{O}\left(10^{-4}\right)$. This fact implies that $H$ and $N_{R}$ almost decouple from the SM sector because $H$ consists mainly of $S$. The SM Higgs boson $h$ can decay into a pair of three light particles. However, the decay widths of $h$ into $H H, N_{R} N_{R}$, and $X X$ pairs are

$$
\begin{array}{r}
\Gamma\left(h \rightarrow N_{R} N_{R}\right)=6.21 \times 10^{-7} \mathrm{MeV}, \\
\Gamma(h \rightarrow H H)=1.03 \times 10^{-3} \mathrm{MeV}, \\
\Gamma(h \rightarrow X X)=1.02 \times 10^{-3} \mathrm{MeV},
\end{array}
$$

for the above reference values. Therefore, the contribution to the invisible Higgs decay width is negligible.

\section{DARK MATTER}

The thermal relic abundance of the lightest right-handed neutrino $N_{R}$ dark matter is obtained by integrating the Boltzmann equation for its number density $n$,

$$
\frac{d n}{d t}+3\left(\frac{\dot{a}(t)}{a(t)}\right) n=-\langle\sigma v\rangle\left(n^{2}-n_{\mathrm{EQ}}^{2}\right),
$$

where $a(t)$ is the scale factor of the expanding Universe, the dot denotes the derivative with respect to time, $\langle\sigma v\rangle$ is the 
(a)

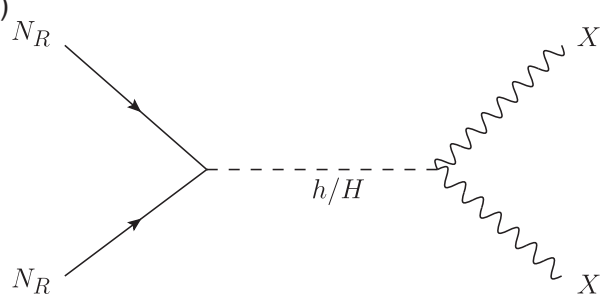

(c)

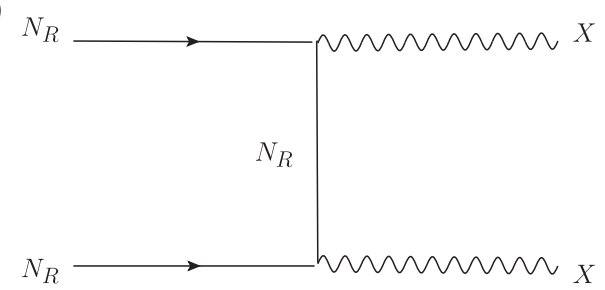

(b)

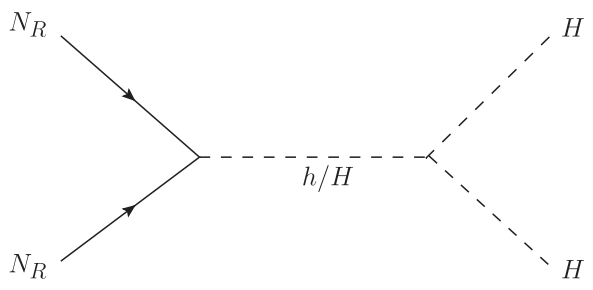

(d)

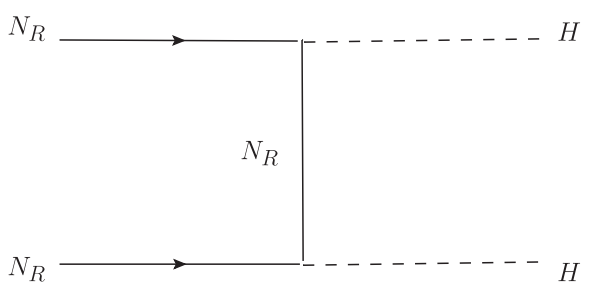

FIG. 1. The rh neutrino annihilation channels into $X X$ and $H H$.

thermal averaged annihilation cross section times relative velocity, and $n_{\mathrm{EQ}}$ is the dark matter number density in thermal equilibrium, respectively.

The lightest rh neutrino dominantly annihilates into pairs of the $X$ boson and the second Higgs boson $H$ via s-channels $H$ exchange shown in Figs. 1(a) and 1(b). Annihilation cross section into $X$ and $H$ bosons pair through $\mathrm{t}(\mathrm{u})$ channels of the $N_{R}$ exchange in Figs. 1(c) and $1(\mathrm{~d})$ is $10^{5}$ times smaller than that of the s-channel $H$ exchange and hence, negligible. Other annihilation modes into the SM fermions through the s-channel exchange of the Higgs bosons ( $h$ and $H$ ) and the $X$ gauge boson [37] are less important, we have included those modes in our numerical calculation nevertheless. We note formula for those annihilation modes in the Appendix for information. The dominant annihilation mode is $N_{R} N_{R} \rightarrow$ $X X$ for a large parameter region. With suggested couplings constant values shown in Eq. (19a), we obtain

$$
m_{\mathrm{DM}} \simeq 3.4 \mathrm{GeV}
$$

in a heavy $H$ cases $\left(m_{H} \gtrsim 9 \mathrm{GeV}\right)$ and which is the maximum dark matter mass in our model. Figure 2 shows the thermal relic abundance of the lightest right-handed neutrino $\Omega_{N_{R}} h^{2}$ in terms of $m_{N_{R}}$. The orange line indicates $\Omega_{\mathrm{DM}} h^{2} \simeq 0.12$ as measured by the Planck satellite [38]. The blue and green curves are for $m_{H}=9 \mathrm{GeV}$ and $2 \mathrm{GeV}$, respectively, as reference values. For light $m_{H}$, lighter DM mass regions also become viable due to features depend on $m_{H}$. Although typical dark matter abundance is large for $m_{\text {DM }}<3.4 \mathrm{GeV}$, even in such a region, the relic abundance is significantly reduced by the resonant annihilation for $m_{N_{R}}=m_{H} / 2$ and can meet with its observed value. This annihilation appears as the deep and narrow gaps in the abundance, which can be seen in the figure. The other characteristic appears at the $m_{N_{R}}=m_{H}$ where the annihilation channel into a $H H$ pair as shown in Fig. 1(b) is kinematically open, and it dominates the annihilation cross section. The sudden decrease of the relic abundance on the left side is explained by this mode.

Thus, one can understand from the figure that the dark matter abundance can be reproduced for the two cases of $m_{N_{R}}$ : (1) at just below the threshold of the $H$ pair annihilation and (2) at both sides of the $H$ resonance or at the off resonance. In the former case, the dark matter mass is determined by

$$
m_{\mathrm{DM}} \simeq m_{H} .
$$

In the Fig. 2, the dark matter mass is obtained as

$$
m_{\mathrm{DM}}=2 \mathrm{GeV},
$$

for $m_{H}=2 \mathrm{GeV}$. As $m_{N_{R}}$ as well as $m_{H}$ are heavy, the annihilation cross section becomes larger and hence, the dark matter abundance can not be explained for $m_{H}=9 \mathrm{GeV}$. In the latter case, the dark matter mass is given by

$$
m_{\mathrm{DM}}^{ \pm}=\frac{m_{H}}{2} \pm \delta m
$$

where the mass difference $\delta m$ is typically from $0.5 \mathrm{GeV}$ $\left(m_{H}=2 \mathrm{GeV}\right)$ to $1 \mathrm{GeV}\left(m_{H}=9 \mathrm{GeV}\right)$. For $m_{H}=9 \mathrm{GeV}$, the dark matter mass is determined as

$$
m_{\mathrm{DM}}^{-} \sim 3.4 \mathrm{GeV}
$$

while for $m_{H}=2 \mathrm{GeV}$,

$$
m_{\mathrm{DM}}^{ \pm}=0.5 \quad \text { or } \quad 1.5 \mathrm{GeV} \text {. }
$$

We note that the results are independent from $\sin \alpha$ as its main annihilation mode are. One may notice that those 
OSAMU SETO and TAKASHI SHIMOMURA

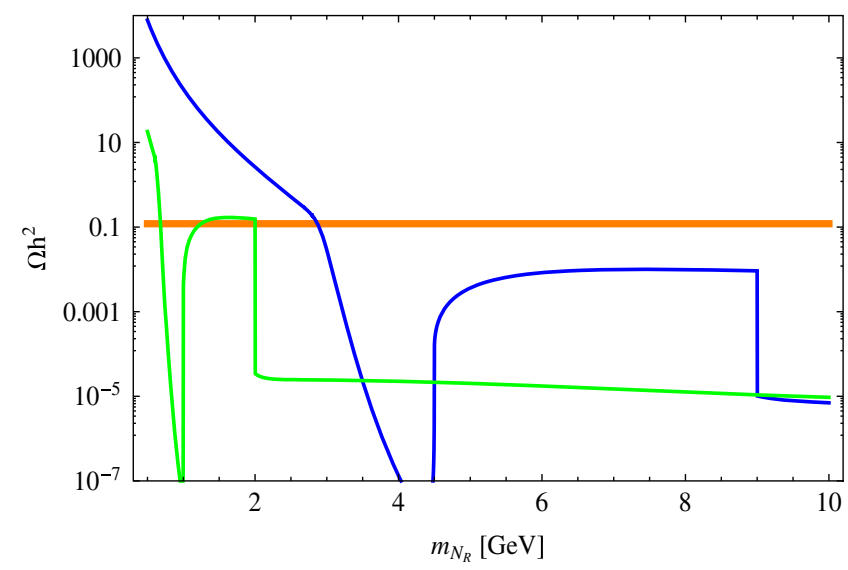

FIG. 2. The right-handed neutrino dark matter thermal relic abundance. The blue and green lines are for $m_{H}=9 \mathrm{GeV}$ and $2 \mathrm{GeV}$, respectively. The orange line indicates $\Omega_{\mathrm{DM}} h^{2} \simeq 0.12$ as measured by Planck satellite.

annihilation processes have a tiny s-wave component and are dominantly p-wave.

This $N_{R}$ dark matter can be searched through the elastic scattering off with a nucleon. The spin-independent scattering cross section with a proton through Higgs bosons exchange is given by [39]

$$
\sigma^{\mathrm{SI}}=\frac{4}{\pi}\left(\frac{m_{p} m_{N_{R}}}{m_{p}+m_{N_{R}}}\right)^{2} f_{p}^{2},
$$

with the proton mass $m_{p}$ and the effective spin-independent coupling between $N_{R}$ and a proton, $f_{p}$, which is given as

$$
\frac{f_{p}}{m_{p}}=\sum_{q=u, d, s} f_{T q}^{(p)} \frac{\alpha_{q}}{m_{q}}+\frac{2}{27} f_{T G}^{(p)} \sum_{c, b, t} \frac{\alpha_{q}}{m_{q}},
$$

where $m_{q}$ is a quark mass, $f_{T q}^{(p)}$ and $f_{T G}^{(p)}$ are constants. The effective vertices between $N_{R}$ and a quark also have been derived in Ref. [37] as

$$
\alpha_{q}=-\frac{m_{N} m_{q}}{v_{s} v} \sin \alpha \cos \alpha\left(\frac{1}{m_{h}^{2}}-\frac{1}{m_{H}^{2}}\right) .
$$

Figure 3 displays the prediction of $\sigma^{\mathrm{SI}}$. We have searched points satisfying $\Omega_{N_{R}} h^{2} \simeq 0.1$ by varying the masses of dark matter $m_{N_{R}}$ and mediator $m_{H}$. The red (blue) points show the results for $\sin \alpha=1 \times 10^{-4}\left(1 \times 10^{-5}\right)$. Here, we have scanned $m_{H}$ for $2 \mathrm{GeV} \leq m_{H} \leq 10 \mathrm{GeV}$, which is enough to find mass range of dark matter. The lower bound on $m_{H}$ we took is due to the following reason. The radiative correction to $\lambda_{s}$ from one loop diagram propagating $N_{R}$ is $\delta \lambda_{s} \simeq-\left(1 / 4 \pi^{2}\right) \sum Y_{R}^{4}$. Provided that the largest coupling of $Y_{R}$ is the order of unity, as we often think from the viewpoint of "naturalness", then we have $\delta \lambda_{s}=\mathcal{O}(-0.01)$. Thus, by considering such radiative corrections, $\lambda_{s}=0.01$ seems to be a sensible lower value and its corresponding

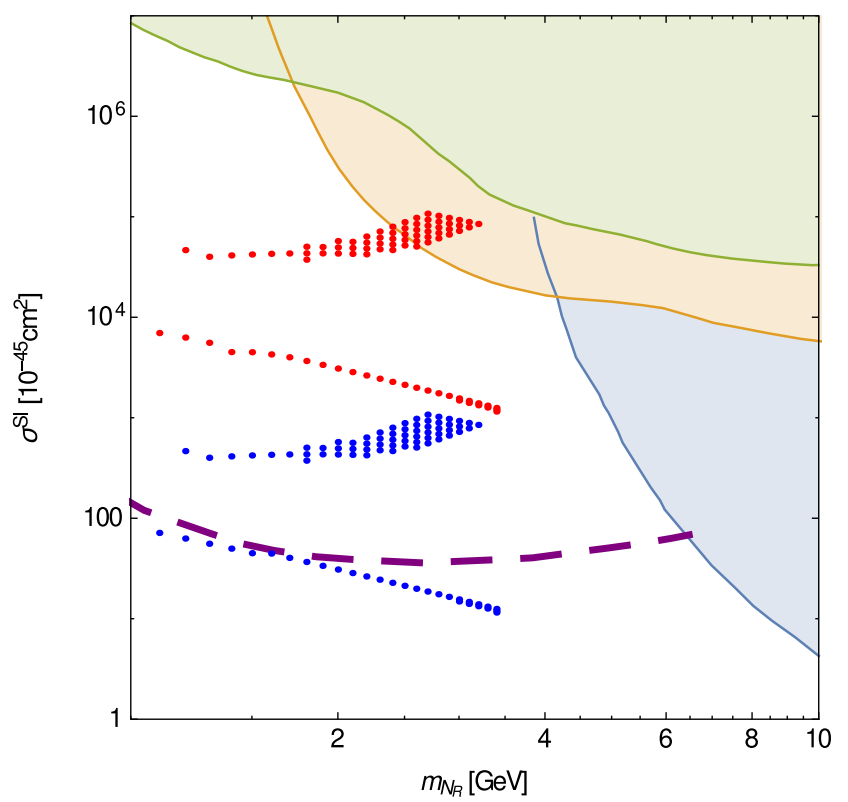

FIG. 3. The spin-independent scattering cross section with a proton. The red (blue) points are for $\sin \alpha=1 \times 10^{-4}\left(1 \times 10^{-5}\right)$. The excluded regions by null results in the CREEST-II, the CDMSlite, and the LUX have shading with green, orange, and blue, respectively. The purple dashed line indicates the expected sensitivity of Ge HV detector in the superCDMS SNOWLAB.

Higgs boson mass estimated from Eq. (26a) is about $2 \mathrm{GeV}$. We show excluded regions by direct dark matter searches, the CREEST-II [40], the CDMSlite [41], and the LUX $[42,43]$. For both red and blue points, one can see two groups of points; the upper group predicting a larger cross section with a nucleon and the lower group predicting a smaller cross section. The former corresponds to the case of Eq. (31) and $m_{\mathrm{DM}}^{+}$in Eq. (33) and the later does to the case of Eq. (34) and $m_{\mathrm{DM}}^{-}$in Eq. (33). The projected sensitivity of the SuperCDMS SNOLAB [44] can cover the predicted regions of $\sin \alpha>\mathcal{O}\left(10^{-5}\right)$.

As we mentioned above, among the dominant annihilation into $X$ bosons pair, only t-(u-) channel $N_{R}$ exchange contribution gives a small s-wave mode of $\mathcal{O}\left(10^{-5}\right) \mathrm{pb}$. Hence, the bounds from dark matter indirect searches such as the Fermi-LAT [45] do not constrain a parameter region of interest in this model. It has been pointed out that, in light of AMS-02 data [46], a low mass region $\left(m_{\mathrm{DM}}<\mathcal{O}(0.1) \mathrm{GeV}\right)$ of dark matter annihilating into electrons is stringently constrained [47]. Even with such a small annihilation cross section, it is not trivial to confirm that this constraint is satisfied for a light mass region. Thus, we restrict investigated dark matter mass range at $\mathrm{GeV}$ region in this paper, we might study such light region elsewhere.

\section{SUMMARY}

Motivated by the Atomki anomalies and nonvanishing neutrino masses, we have considered a gauged $U(1)_{B-L}$ 
extended radiative seesaw model. We showed that the anomalies as well as the dark matter abundance and nonvanishing neutrino masses can be explained simultaneously.

Requiring the decay of the $B-L$ gauge boson to be the origin of the Atomki anomalies, we showed that the model parameters can be determined or constrained. The resulting mass of the lightest right-handed neutrino dark matter is below about $3.4 \mathrm{GeV}$ and that of the lighter $Z_{2}$ even scalar is also below about $20 \mathrm{GeV}$ assuming the coupling to be smaller than the unity. However, such light particles must almost decouple from the SM due to small couplings. Therefore, the Higgs sector remains the SM-like, which is consistent with the LHC results.

We have also found that the relic dark matter abundance can be reproduced by the annihilation into $X X$. It further constrains the scalar mixing angle and the dark matter mass. We have shown the consistent model parameter region with $\Omega_{N_{R}} h^{2} \simeq 0.1$, where the elastic scattering cross section of the DM particle off nuclei can be below the current bound from the CRESST-II, the CDMSlite, and LUX experiments. However, the cross section is predicted within the reach of the expected sensitivity of Ge $\mathrm{HV}$ detector. Therefore, our dark matter candidate is in practice detectable even for an extremely small Higgs mixing angle $\sin \alpha$.

\section{ACKNOWLEDGMENTS}

T. S would like to thank Y. Maeda for fruitful discussion on nuclear experiments. We are grateful for M. Aoki's valuable comments letting us notice an error in the previous calculation. This work is supported, in part, by JSPS KAKENHI Grants No. 15K17654 (T. S) and No. 26400243 (O. S.) and by the SUHARA Memorial Foundation (O. S.).

\section{APPENDIX: AMPLITUDE}

We give explicit formulas of the invariant amplitude squared for the pair annihilation processes of the rh neutrinos.

\section{Annihilation into $X X$}

$\mathcal{M}_{1}$ denotes the amplitude by the $s$-channel Higgs bosons $h$ and $H$ exchange, while $\mathcal{M}_{2}$ does that for the $t(u)$-channel $N$ exchange diagram.

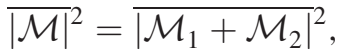

$$
\begin{aligned}
& {\overline{\mid \mathcal{M}_{1}}}^{2}=m_{N}^{2} q_{B-L}^{2} g_{B-L}^{2}\left|\sin ^{2} \alpha \frac{1}{s-m_{h}^{2}+i m_{h} \Gamma_{h}}+\cos ^{2} \alpha \frac{1}{s-m_{H}^{2}+i m_{H} \Gamma_{H}}\right|^{2} \times\left(s-4 m_{N}^{2}\right)\left(1+\frac{1}{2 m_{X}^{4}}\left(\frac{s}{2}-m_{X}^{2}\right)^{2}\right), \\
& \int \frac{d \cos \theta}{2} \overline{\left|\mathcal{M}_{2}\right|^{2}}=\frac{32 g_{B-L}^{4} q_{B-L}^{4}}{m_{X}^{4}}\left(\frac{4 m_{N}^{4} s\left(s-4 m_{X}^{2}\right)+4 m_{N}^{2} m_{X}^{2}\left(4 m_{X}^{2}-s\right)\left(m_{X}^{2}+s\right)-m_{X}^{4}\left(4 m_{X}^{4}+s^{2}\right)}{\sqrt{s-4 m_{N}^{2}} \sqrt{s-4 m_{X}^{2}}\left(s-2 m_{X}^{2}\right)}\right. \\
& \left.\times \ln \left[\frac{s-2 m_{X}^{2}-\sqrt{s-4 m_{N}^{2}} \sqrt{s-4 m_{X}^{2}}}{s-2 m_{X}^{2}+\sqrt{s-4 m_{N}^{2}} \sqrt{s-4 m_{X}^{2}}}\right]+\frac{2 m_{N}^{4}\left(8 m_{X}^{4}-8 m_{X}^{2} s+s^{2}\right)+m_{N}^{2} m_{X}^{4}\left(4 m_{X}^{2}+s\right)-2 m_{X}^{8}}{\left(m_{N}^{2}\left(s-4 m_{X}^{2}\right)+m_{X}^{4}\right)}\right), \\
& \int \frac{d \cos \theta}{2} \overline{\left(\mathcal{M}_{1} \mathcal{M}_{2}^{*}+c . c\right)}=\frac{8 \sqrt{2} g_{B-L}^{4} q_{B-L}^{4} v_{s} \lambda m_{N}}{m_{X}^{4} \sqrt{s-4 m_{N}^{2}} \sqrt{s-4 m_{X}^{2}}}\left(\frac{-\sin \alpha\left(m_{h}^{2}-s\right)}{\Gamma_{h}^{2} m_{h}^{2}+\left(m_{h}^{2}-s\right)^{2}}+\frac{\cos \alpha\left(m_{H}^{2}-s\right)}{\Gamma_{H}^{2} m_{H}^{2}+\left(m_{H}^{2}-s\right)^{2}}\right) \\
& \times\left(\sqrt{s-4 m_{N}^{2}}\left(4 m_{X}^{4}-2 m_{X}^{2} s+s^{2}\right) \sqrt{s-4 m_{X}^{2}}+2\left(m_{N}^{2}\left(8 m_{X}^{4}-4 m_{X}^{2} s+s^{2}\right)-2 m_{X}^{6}\right)\right. \\
& \left.\times \log \left[\frac{s-2 m_{X}^{2}-\sqrt{s-4 m_{N}^{2}} \sqrt{s-4 m_{X}^{2}}}{s-2 m_{X}^{2}+\sqrt{s-4 m_{N}^{2}} \sqrt{s-4 m_{X}^{2}}}\right]\right),
\end{aligned}
$$

where $\theta$ is the scattering angle in the center of mass frame.

\section{Annihilation into $\mathrm{HH}$}

$\mathcal{M}_{1}$ denotes the amplitude by the $s$-channel Higgs bosons $h$ and $H$ exchange, while $\mathcal{M}_{2}$ does that for the $t(u)$-channel $N$ exchange diagram.

$$
\overline{\mid \mathcal{M}}^{2}=\overline{\left|\mathcal{M}_{1}+\mathcal{M}_{2}\right|^{2}}
$$




$$
\begin{aligned}
& \left.\overline{\mid \mathcal{M}_{1}}\right|^{2}=\frac{\lambda_{N}^{2}}{4}\left(s-4 m_{N}^{2}\right)\left|\frac{\sin \alpha}{s-m_{h}^{2}+i m_{h} \Gamma_{h}} \lambda_{h H H}-\frac{\cos \alpha}{s-m_{H}^{2}+i m_{H} \Gamma_{H}} \lambda_{H H H}\right|^{2}, \\
& \int \frac{d \cos \theta}{2}\left|\overline{\mathcal{M}_{2}}\right|^{2}=\frac{\lambda_{N}^{4}}{2} \cos ^{4} \alpha\left(-8-\frac{4\left(m_{H}^{2}-4 m_{N}^{2}\right)^{2}}{m_{H}^{4}-4 m_{H}^{2} m_{N}^{2}+m_{N}^{2} s}+4 \frac{\left(6 m_{H}^{4}-32 m_{N}^{4}+16 m_{N}^{2} s+s^{2}-4 m_{H}^{2}\left(4 m_{N}^{2}+s\right)\right)}{\left(s-2 m H^{2}\right) \sqrt{\left(s-4 m_{N}^{2}\right)\left(s-4 m_{H}^{2}\right)}}\right. \\
& \left.\times \ln \left[\frac{s-2 m_{H}^{2}+\sqrt{\left(s-4 m_{N}^{2}\right)\left(s-4 m_{H}^{2}\right)}}{s-2 m_{H}^{2}-\sqrt{\left(s-4 m_{N}^{2}\right)\left(s-4 m_{H}^{2}\right)}}\right]\right) \\
& \int \frac{d \cos \theta}{2} \overline{\left(\mathcal{M}_{1} \mathcal{M}_{2}^{*}+c . c\right)}=8 \sqrt{2} \lambda_{N}^{3} m_{N} \cos ^{2} \alpha\left(-\frac{\sin \alpha\left(s-m_{h}^{2}\right) \lambda_{h H H}}{\left(s-m_{h}^{2}\right)^{2}+\left(M_{h} \Gamma_{h}\right)^{2}}+\frac{\cos \alpha\left(s-m_{H}^{2}\right) \lambda_{H H H}}{\left(s-m_{H}^{2}\right)^{2}+\left(M_{H} \Gamma_{H}\right)^{2}}\right) \\
& \times\left(1+\frac{s-8 m_{N}^{2}+2 m_{H}^{2}}{2 \sqrt{\left(s-4 m_{N}^{2}\right)\left(s-4 m_{H}^{2}\right)}} \ln \left[\frac{s-2 m_{H}^{2}+\sqrt{\left(s-4 m_{N}^{2}\right)\left(s-4 m_{H}^{2}\right)}}{s-2 m_{H}^{2}-\sqrt{\left(s-4 m_{N}^{2}\right)\left(s-4 m_{H}^{2}\right)}}\right]\right) .
\end{aligned}
$$

[1] P. Minkowski, Phys. Lett. 67B, 421 (1977).

[2] T. Yanagida, Conference Proceedings C7902131, 95 (1979).

[3] M. Gell-Mann, P. Ramond, and R. Slansky, Conference Proceedings C790927, 315 (1979).

[4] R. N. Mohapatra and G. Senjanovic, Phys. Rev. Lett. 44, 912 (1980).

[5] W. Konetschny and W. Kummer, Phys. Lett. 70B, 433 (1977).

[6] T. P. Cheng and L.-F. Li, Phys. Rev. D 22, 2860 (1980).

[7] J. Schechter and J. W. F. Valle, Phys. Rev. D 22, 2227 (1980).

[8] R. Foot, H. Lew, X. G. He, and G. C. Joshi, Z. Phys. C 44, 441 (1989).

[9] A. Zee, Phys. Lett. 93B, 389 (1980); 95B, 461(E) (1980).

[10] A. Zee, Nucl. Phys. B264, 99 (1986).

[11] K. S. Babu, Phys. Lett. B 203, 132 (1988).

[12] L. M. Krauss, S. Nasri, and M. Trodden, Phys. Rev. D 67, 085002 (2003).

[13] E. Ma, Phys. Rev. D 73, 077301 (2006).

[14] A. J. Krasznahorkay et al., Phys. Rev. Lett. 116, 042501 (2016).

[15] J. L. Feng, B. Fornal, I. Galon, S. Gardner, J. Smolinsky, T. M. P. Tait, and P. Tanedo, Phys. Rev. Lett. 117, 071803 (2016).

[16] J. L. Feng, B. Fornal, I. Galon, S. Gardner, J. Smolinsky, T. M. P. Tait, and P. Tanedo, Phys. Rev. D 95, 035017 (2017).

[17] P.-H. Gu and X.-G. He, Nucl. Phys. B919, 209 (2017).

[18] M. J. Neves and J. A. Helayel-Neto, arXiv:1609.08471.

[19] Y. Kahn, G. Krnjaic, S. Mishra-Sharma, and T. M. P. Tait, J. High Energy Phys. 05 (2017) 002.

[20] U. Ellwanger and S. Moretti, J. High Energy Phys. 11 (2016) 039.
[21] L.-B. Jia and X.-Q. Li, Eur. Phys. J. C 76, 706 (2016).

[22] T. Kitahara and Y. Yamamoto, Phys. Rev. D 95, 015008 (2017).

[23] C.-S. Chen, G.-L. Lin, Y.-H. Lin, and F. Xu, arXiv:1609.07198.

[24] S. Kanemura, O. Seto, and T. Shimomura, Phys. Rev. D 84, 016004 (2011).

[25] T. Li and W. Chao, Nucl. Phys. B843, 396 (2011).

[26] S. Khalil, H. Okada, and T. Toma, J. High Energy Phys. 07 (2011) 026.

[27] M. Lindner, D. Schmidt, and T. Schwetz, Phys. Lett. B 705, 324 (2011).

[28] S. Kanemura, T. Nabeshima, and H. Sugiyama, Phys. Rev. D 85, 033004 (2012).

[29] H. Okada and T. Toma, Phys. Rev. D 86, 033011 (2012).

[30] S. Kanemura, T. Nabeshima, and H. Sugiyama, Phys. Rev. D 87, 015009 (2013).

[31] Y. Kajiyama, H. Okada, and K. Yagyu, Nucl. Phys. B874, 198 (2013).

[32] Y. Kajiyama, H. Okada, and T. Toma, Phys. Rev. D 88, 015029 (2013).

[33] T. Basak and T. Mondal, Phys. Rev. D 89, 063527 (2014).

[34] S. Kanemura, T. Matsui, and H. Sugiyama, Phys. Rev. D 90, 013001 (2014).

[35] H. Okada and Y. Orikasa, Phys. Lett. B 760, 558 (2016).

[36] W. Wang and Z.-L. Han, Phys. Rev. D 92, 095001 (2015).

[37] N. Okada and O. Seto, Phys. Rev. D 82, 023507 (2010).

[38] P. A. R. Ade et al. (Planck Collaboration), Astron. Astrophys. 594, A13 (2016).

[39] G. Jungman, M. Kamionkowski, and K. Griest, Phys. Rep. 267, 195 (1996).

[40] G. Angloher et al. (CRESST-II Collaboration), Eur. Phys. J. C 74, 3184 (2014). 
[41] R. Agnese et al. (SuperCDMS Collaboration), Phys. Rev. Lett. 116, 071301 (2016).

[42] D. S. Akerib et al. (LUX Collaboration), Phys. Rev. Lett. 116, 161301 (2016).

[43] D. S. Akerib et al. (LUX Collaboration), Phys. Rev. Lett. 118, 021303 (2017).

[44] R. Agnese et al., Phys. Rev. D 95, 082002 (2017).
[45] M. L. Ahnen et al. (Fermi-LAT Collaboration, MAGIC Collaboration), J. Cosmol. Astropart. Phys. 02 (2016) 039.

[46] M. Aguilar et al. (AMS Collaboration), Phys. Rev. Lett. 110, 141102 (2013).

[47] A. Ibarra, A. S. Lamperstorfer, and J. Silk, Phys. Rev. D 89, 063539 (2014). 